\title{
Sleep loss and circadian disruption in shift work: health burden and management
}

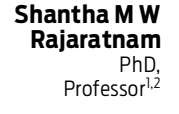

Mark E Howard MB BS, FRACP, PhD Director $^{3}$

Ronald R Grunstein FRACP, MD, PhD, Professor of Sleep Medicine and NHMRC Practitione

Fellow, ${ }^{2}$ and Senior Staff Specialist

1School of Psychology and Psychiatry,

Monash University,

Melbourne, VIC

2 NHMRC Centre for Integrated Research and Understanding of Sleep, Sydney, NSW.

3 Victorian Respiratory Support Service. Austin Health, Melbourne, VIC.

4 Respiratory and Sleep Medicine, Royal Prince Alfred Hospital Sydney, NSW.

shantha.rajaratnam@ monash.edu

MJA 2013; 199: SII-S15 doi: 10.5694/mjal3.1056

Online first 17/10/13 early 1.5 million Australians are employed in shift work, representing $16 \%$ of the working population. Shift work is associated with adverse health, safety and performance outcomes. Circadian rhythm misalignment, inadequate and poor-quality sleep, and sleep disorders are thought to contribute to these associations.

The most immediate consequence of shift work is impaired alertness, which has widespread effects on core brain functions - reaction time, decision making, information processing and the ability to maintain attention. This impairment leads to preventable errors, accidents and injuries, especially in high-risk environments. Long-term health consequences of shift work have been reported, including increased vascular events. ${ }^{1}$

This review evaluates the health burden associated with shift work and discusses strategies for the clinical management of sleep-wake disturbances in shift workers. Evidence-based management strategies require consideration of the key physiological sleep-wake determinants of alertness (Box 1).

\section{Circadian and sleep-wake disturbances}

\section{Circadian timing}

The endogenous circadian pacemaker located in the hypothalamic suprachiasmatic nuclei generates and maintains the timing of behavioural and physiological events according to a 24-hour rhythm. The pacemaker signals increased alertness during the day and high sleep propensity at night. Night shift and rotating or extended-duration shifts involve working at the time of the circadian nadir, when sleep propensity is maximal and consequently alertness is substantially impaired. Often complete circadian adaptation does not occur even in permanent night shift workers ${ }^{2}$ and, as a result, many night workers experience misalignment of their circadian pacemaker and the imposed sleep-wake cycle. The effects of this misalignment are exacerbated by chronic sleep restriction (see below) due to insomnia and reduced sleep duration during the day. ${ }^{2}$ Misalignment between the circadian pacemaker and the sleep-wake cycle may result in shift work disorder, defined as insomnia during daytime sleep and/or excessive sleepiness during wake episodes temporally associated with the shift schedule and occurring for at least 1 month. ${ }^{3}$

Circadian modulation of several cardiovascular risk markers (eg, circulating cortisol and catecholamines, blood pressure, cardiac vagal modulation) has been described, consistent with epidemiological studies showing a peak in adverse cardiovascular events in the morning. ${ }^{4}$ Recent laboratory studies demonstrate that circadian misalignment (such that individuals sleep 12 hours out of phase with the circadian pacemaker) leads to impaired cardiovascular and metabolic function - for example, decreased leptin levels, increased glucose levels despite increased

\section{Summan}

- About 1.5 million Australians are shift workers. Shift work is associated with adverse health, safety and performance outcomes. Circadian rhythm misalignment, inadequate and poor-quality sleep, and sleep disorders such as sleep apnoea, insomnia and shift work disorder (excessive sleepiness and/or insomnia temporally associated with the work schedule) contribute to these associations.

- Falling asleep at work at least once a week occurs in 32\%-36\% of shift workers. Risk of occupational accidents is at least $60 \%$ higher for non-day shift workers. Shift workers also have higher rates of cardiometabolic diseases and mood disturbances.

- Road and workplace accidents related to excessive sleepiness, to which shift work is a significant contributor, are estimated to cost \$71-\$93 billion per annum in the United States.

- There is growing evidence that understanding the interindividual variability in sleep-wake responses to shift work will help detect and manage workers vulnerable to the health consequences of shift work.

- A range of approaches can be used to enhance alertness in shift workers, including screening and treating sleep disorders, melatonin treatment to promote sleep during the daytime, and avoidance of inappropriate use of sedatives and wakefulness-promoters such as modafinil and caffeine. Short naps, which minimise sleep inertia, are generally effective.

- Shifting the circadian pacemaker with appropriately timed melatonin and/or bright light may be used to facilitate adjustment to a shift work schedule in some situations, such as a long sequence of night work.

- It is important to manage the health risk of shift workers by minimising vascular risk factors through dietary and other lifestyle approaches.

insulin levels, reversed daily cortisol rhythm and increased mean arterial pressure. ${ }^{4}$

\section{Duration of wakefulness}

With increasing duration of wakefulness, the propensity for sleep increases and alertness becomes impaired. In an individual with a healthy sleep-wake cycle, alertness is maintained at a relatively stable level through interactions between the circadian pacemaker and the system that tracks how long the individual has been awake, referred to as the sleep homoeostat. ${ }^{2}$ After about 16 hours, alertness will sharply decline such that the magnitude of impairment in neurobehavioural performance after 17 hours of wakefulness is comparable to that observed at a blood alcohol concentration of $0.05 \% .{ }^{5}$ After 24 hours of sleep deprivation, performance impairment is similar in magnitude to that observed at a blood alcohol concentration of $0.10 \%$. 


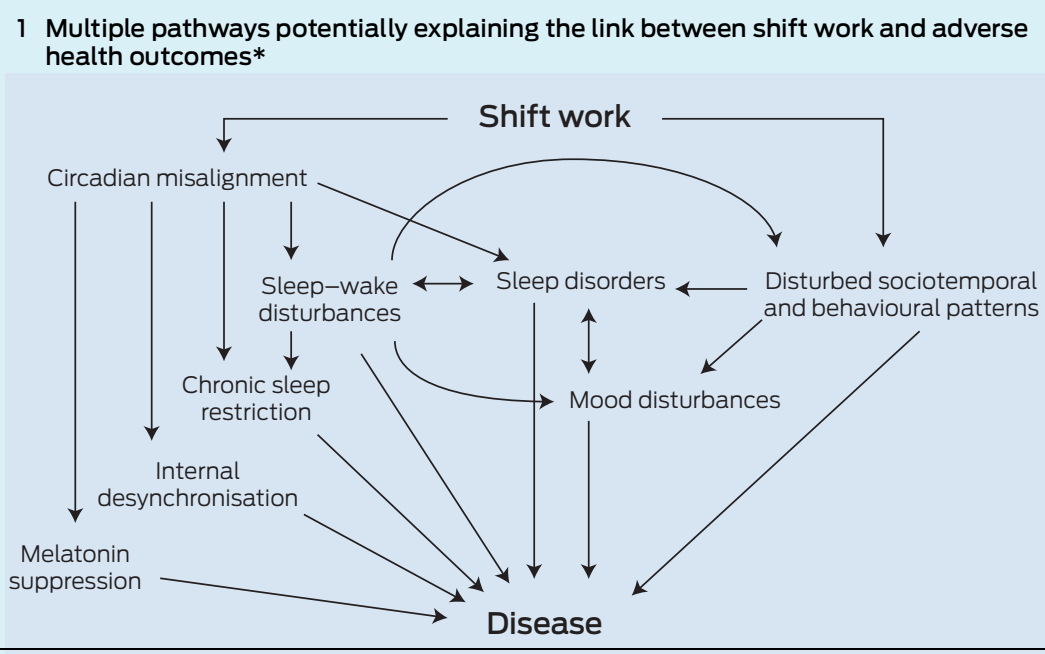

* Modified with permission from Knutsson A. Health disorders of shift workers. Occup Med (Lond) 2003; 53: 103-108.

\section{Sleep duration}

In laboratory studies, duration of the sleep episode shows a dose-dependent relationship with daytime neurobehavioural performance, ${ }^{6}$ reflecting the adverse impact of chronic sleep restriction on alertness level. Adverse effects of chronic sleep restriction on cardiometabolic outcomes have also been demonstrated in both laboratory and epidemiological studies. ${ }^{7}$ Although variations in intrinsic sleep need in the general population are well recognised, lifestyle factors appear to explain a substantial proportion of the variation in habitual sleep duration. ${ }^{8}$ Poor sleep quality due to a sleep disorder, other medical conditions or misalignment of sleep in shift workers results in chronic sleep restriction, which causes an even greater degree of alertness impairment overnight in shift workers. ${ }^{9}$

\section{Sleep disorders}

Alertness impairment is a hallmark symptom of many sleep disorders. Disorders such as obstructive sleep apnoea (OSA), insomnia and shift work disorder are associated with performance impairment or lost productivity, and increased risk of motor vehicle crashes and occupational injuries. ${ }^{6,10}$ Sleep disorders are more common among shift workers, exacerbating the risk of adverse safety, performance and health outcomes. A recent large survey of a broad range of Australian workers found that 32\% of night workers suffered from shift work disorder, including 9\% with a severe problem. ${ }^{11}$ Among United States police officers, $40.1 \%$ screened positive on a survey for at least one sleep disorder, with the most common being OSA (33.6\%), followed by moderate to severe insomnia (6.5\%), shift work disorder $(5.4 \%$ of total, or $14.5 \%$ of those who work night shifts), restless legs syndrome (1.6\%) and narcolepsy with cataplexy $(0.4 \%) .{ }^{6,10}$

\section{Health and safety burden associated with shift work}

The mismatch between the endogenous circadian pacemaker and the sleep-wake cycle results in immediate sleep-wake disturbances, chronic sleep restriction and possibly internal desynchronisation of the circadian system (Box 1). This results in deleterious effects on alertness, cognitive function, mood, social and work activities, and health. Sleepiness is common, increased by more than $50 \%$ in truck drivers working night shift and associated with brief sleep episodes. ${ }^{12}$ Falling asleep during night shift occurred at least weekly in $36 \%$ of rotating shift workers, $32 \%$ of permanent night workers and $21 \%$ of day and evening nurses working an occasional night shift. ${ }^{13}$

Given this impairment in alertness and cognitive function, it is not surprising that, compared with day workers, the risk of accidents and near-miss events is significantly elevated in shift workers, including those involved in safety-critical industries such as health care, law enforcement and commercial driving. ${ }^{14}$ Major catastrophes such as the industrial accidents at Three Mile Island, Chernobyl and Bhopal have been linked to human error related to shift work. ${ }^{2}$ Shift workers have impaired driving performance and a two to four times increased risk of crashing during their commute to and from work. ${ }^{6,13}$ Sleep-related accidents are most common during the night shift in transportation fields, peaking towards the end of the night shift, and the risk of occupational accidents is also increased when working outside regular daytime hours (relative risk, 1.6). ${ }^{15}$ These findings are consistent with those from the general population showing increased risk of motor vehicle crash during the night and after sleep restriction. ${ }^{16}$ In addition to personal and public safety risks, productivity is impaired, with frequent workplace errors and increased absenteeism. ${ }^{17}$ Conversely, an intervention based on circadian principles significantly improved productivity in rotating shift workers. ${ }^{18}$ There is a marked increase in preventable medical errors, including those resulting in fatalities, when medical residents work frequent extended-duration night shifts. ${ }^{6}$ In police officers, poor sleep associated with shift work is also related to impaired function at work, including administrative and safety errors, falling asleep in meetings, uncontrolled anger and absenteeism. ${ }^{10}$ Hence, shift work can impact on the safety of the worker and others, as well as reducing productivity.

Shift work is associated with a higher risk of several medical conditions, particularly metabolic syndrome, cardiovascular diseases and mood disorders. ${ }^{6}$ Increased cancer risk has also been described, potentially through disruption of the circadian system from light exposure at night. ${ }^{19}$ Circadian misalignment is related to cardiometabolic changes, and together with altered food choice and physical activity, leads to increases in obesity, dyslipidaemia and impaired glucose metabolism. Rotating shift workers are $20 \%-30 \%$ more likely to have impaired glucose metabolism (elevated $\mathrm{HbA}_{1 \mathrm{c}}$ levels) with a $70 \%$ increase in metabolic syndrome among transport workers. ${ }^{20}$ In large epidemiological studies, mortality from diabetes, cardiovascular disease and stroke is higher in longterm shift work, although all-cause mortality is not clearly increased. ${ }^{21}$ Mood disturbance is common during rotating and night shifts, although the longer-term effects of shift work on mood are less clear. Doctors experience symptoms of anxiety, depressed mood and reduced motivation, in conjunction with impaired cognition, during prolonged 
night shifts. ${ }^{22}$ A recent US study of police found that anxiety and depression were more than twice as common in those who had symptoms of disordered sleep. ${ }^{10}$ Depressive symptoms in shift workers are also linked to increased absenteeism and occupational errors. ${ }^{23}$ Although impaired mood is common during shift cycles, it remains unclear as to whether shift work results in longer-term mood disturbance.

The health and economic costs of shift work-related sleep-wake disturbances are high, taking into account the combined effects of impaired sleep, workplace and road accidents, mood disorders, lost productivity and cardiovascular health. Precise economic costs have not been quantified, although the economic impact of individual elements provides some idea. The average cost per year to a person suffering from regular insomnia, as occurs with shift work, is estimated at over $\$ 5000$. Excessive sleepiness occurs in more than $30 \%$ of shift workers. The combined cost of road and workplace accidents caused by excessive sleepiness is estimated for 2009 at \$71-\$93 billion per annum in the US, with shift work a major contributor to this cost. ${ }^{24}$

\section{Clinical management of circadian and sleep- wake disturbances in shift workers}

The key aims of managing sleep-wake problems in shift workers are to ensure sustained alertness during wake episodes when working and during social activities, and to facilitate restorative sleep when sleep is required. In part, this is achieved by prevention or minimisation of factors that worsen sleep-wake function and therefore impair alertness, such as long work hours or rotating shift schedules, an approach that has been shown to reduce adverse events related to shift work in the health sector. Forward rotation of shifts (from day to afternoon to night) is preferable. Second, given the interindividual variability in sleep-wake responses to shift work, it is also important to develop algorithms that predict whether a shift worker is fit for duty or potentially vulnerable to alertness failure. There have been major efforts to develop biomathematical models using information such as work and sleep-wake schedules to evaluate safety risk associated with particular shift rosters. The use of such approaches outside of the research setting is considered premature. In high-risk industries, such as transportation, companies should have systems in place to minimise the risk related to shift work.

With shift work, sleep-wake disorders are highly probable at some stage in all workers, and an approach to mitigate the consequences of shift work should be adopted in the workplace as occupational health policy; for example, through screening programs for sleep disorders and general health.

\section{Managing sleep complaints}

It is important to identify and address any comorbid conditions that independently cause insomnia or sleepiness further compromising alertness in the shift worker. Examples are conditions such as OSA, or mood disorders such as depression. Treatment of acute or chronic insomnia is important to maintain sleep continuity, adequate sleep length and alertness during wakefulness. Psychological approaches such as cognitive behavioural therapy are important in managing chronic insomnia. ${ }^{25}$ Limited but judicious use of sedative-hypnotic medication may help workers adapt to rotating sleep schedules but the data are controversial. Sedative-hypnotics should be used carefully owing to their potential side effects, including the carryover of sedation to the night shift, which may negatively affect performance and safety. ${ }^{26}$ Use of alcohol, cannabis and non-medically prescribed drugs to manage sleep complaints should be discouraged.

\section{Pharmacotherapy to improve alertness}

The reality of shift work and long schedules during sustained operations, particularly in the transport industry, has resulted in even regulators considering use of medications to promote alertness under certain situations. This medicalisation of shift work to prevent human error and resulting consequences is controversial. Use of stimulants such as ephedrine or amphetamine is illegal and stigmatised. However, the availability of wakefulness-promoting agents modafinil and armodafinil (the R-enantiomer of modafinil), which improve alertness compared with placebo without much of the adverse effect profile of stimulants, has resulted in the possibility of managing alertness failure during shift work through pharmacotherapy. Based on evidence from large controlled clinical trials, ${ }^{27}$ these agents are now specifically approved by the US Food and Drug Administration for the treatment of excessive sleepiness in workers with shift work disorder. Self-limiting headache is the most commonly reported adverse event with these drugs.

Caffeine is used universally as a stimulant to maintain alertness. Caffeine improves cognitive performance in shift workers. ${ }^{28}$ A variety of doses, preparations and administration regimens are reported to be effective, ${ }^{28}$ including a single dose of $200 \mathrm{mg}$ and a low-dose, repeated caffeine administration protocol $(0.3 \mathrm{mg} / \mathrm{kg} / \mathrm{h})$. Residual effects of higher doses of caffeine on daytime sleep have been reported, ${ }^{29}$ which should be taken into consideration in caffeine administration guidelines, particularly for alertness management in night shift workers.

It should be noted that the above pharmacological strategies are aimed at managing sleepiness symptoms in shift workers. There is no evidence that these can facilitate circadian adaptation to a shift schedule or promote sleep.

\section{Napping}

Scheduled napping for shift workers may be useful in relieving excessive sleepiness during work shifts. ${ }^{6}$ However, the exact configuration of naps that maximises alertness on duty has yet to be clarified. Naps ranging from 20 to 40 minutes taken during night shifts (eg, between 2 am and $3 \mathrm{am})^{30}$ are beneficial, as is prophylactic napping before a night shift. ${ }^{6}$

The potential for alertness impairment due to sleep inertia should be considered and sufficient time allowed for its dissipation, particularly for naps occurring during work shifts. Sleep inertia refers to the impairment that occurs immediately on awakening and can last from minutes up to several hours. The magnitude of impairment may even be worse than that after 24 hours of sleep deprivation. ${ }^{31}$ The severity of sleep inertia varies according to the stage of sleep and circadian phase from which the 
awakening occurred. There is insufficient evidence to recommend how long an individual in operational settings should wait after a nap for the effects of sleep inertia to dissipate. A recent laboratory-based, simulated night shift work study in healthy male volunteers suggests that a 15minute interval should be allowed following nap opportunities of up to 60 minutes, and also that workplace education be provided that subjective feelings of sleepiness are not a reliable indicator of performance impairments due to sleep inertia. ${ }^{32}$

\section{Light and melatonin for circadian adaptation}

Timed administration of melatonin can facilitate adaptation of the circadian pacemaker to a new sleep-wake schedule ${ }^{33}$ however, this is not recommended for rapidly rotating shift schedules. Melatonin can also be used to promote sleep during the daytime, thereby improving sleep quality and duration in night shift workers. ${ }^{33}$ Although melatonin is safe for short-term use, long-term safety data are lacking.

For adaptation to a series of night shifts, the following is recommended: light exposure in the night and early morning hours to facilitate a circadian phase delay (ie, shift of the circadian pacemaker to a later time) and promote alertness; and shielding morning light exposure to minimise the competing circadian phase advance effect of $\operatorname{light}^{34}$ and to reduce the residual impact of the alerting effect of light on daytime sleep. However, this regimen is only suitable for a limited range of shift types. There appears to be an increase in the frequency of certain types of shift schedule that expose individuals to higher safety risks, including slow rotating, long duration ( $\geqslant 12$ hours) and quick return (a break of only 8 hours when changing from one shift to another) shifts. Thus, the application of light treatment needs to be considered on a case-by-case basis, taking into account the specific characteristics of each schedule. Light is the most potent time cue for the circadian pacemaker, synchronising it to the 24-hour day. The magnitude and direction (ie, shift to an earlier or later time) of the effect critically depend on the timing of the exposure as well as the intensity, duration and wavelength. Here, timing relates to phase of the endogenous circadian pacemaker, which would ideally be measured through assessment of endogenous melatonin levels in saliva or core body temperature levels before an intervention. Timed light and darkness exposure can be used to facilitate adaptation of the circadian pacemaker to a new shift schedule.

\section{Reducing risk of cardiometabolic disease}

Shift workers are at higher risk of cardiometabolic diseases and are therefore targets for closer monitoring of risk factors and avoiding unhealthy diets. High fat meals consumed during the night may produce more postprandial hypertriglyceridaemia than equivalent meals during the day. ${ }^{2}$ Promoting physical activity in the workplace and home is another countermeasure to cardiometabolic risk. Laboratory studies have shown that exercise during the night phase shifts the circadian pacemaker, ${ }^{35}$ thus potentially facilitating biological adaptation to shift work.

Shift work is commonly associated with adverse safety and health consequences (Box 2). Circadian misalignment,

\section{Shift work summary}

- Misalignment between the circadian pacemaker and the timing of sleep, wake and work occurs in shift workers.

- Shift work disorder, with insomnia, reduced sleep and excessive sleepiness, is common.

- These abnormalities impair cognitive function, alertness and mood and increase accident risk.

- Metabolic syndrome is also common in shift workers, resulting in increased cardiovascular risk.

Practical tips for the management of the chronically sleepy (night) shift worker

- Optimal shift schedule is important, allowing adequate time for recovery sleep and minimising extended duration shifts.

- Have at least 7 hours of sleep per 24 hours.

- Initiate main sleep episode as soon as practicable after evening or night shift.

- Nap for 30 minutes to 2 hours before evening or night shifts to supplement main sleep episode.

- Nap for 20-30 minutes during night shift to help maintain wakefulness, particularly for high-risk occupations (eg, driving).

- Keep bedroom quiet and dark, use earplugs.

- Increase exposure to bright light during evening/first half of a night shift.

- After a night shift, avoid exposure to bright light; eg, use sunglasses or blue-light blocking glasses.

- Melatonin (1-2 mg) is effective in promoting daytime sleep.

- Caffeine can be used to promote alertness. High-frequency (eg, hourly) low-dose caffeine administration (eg, 30-40 mg - about one cup of tea or half a cup of instant coffee) is effective. High doses should be avoided close to daytime sleep.

- Novel alertness-enhancing agents may be beneficial in managing shift work disorder.

- Screen for sleep and mood disorders (eg, shift work disorder, sleep apnoea, insomnia, depression).

- Cardiovascular risk factors should also be addressed as a part of the clinical management plan.

sleep loss and sleep disorders all contribute to these risks, and therefore should be the primary targets for clinical management approaches. Improved methods to detect those who are most vulnerable to the effects of shift work are needed. Diagnosis and management of shift work disorder is an important first step in tackling the significant health burden associated with shift work.

Competing interests: Shantha Rajaratnam has served as a consultant through his institution to Vanda Pharmaceuticals, Philips Respironics, EdanSafe, National Transport Commission, Rail, Tram and Bus Union, Australian Workers' Union, Tontine Group, Meda Consumer Healthcare, and has, through his institution, received research grants and unrestricted educational grants from Vanda Pharmaceuticals, Philips Respironics and Cephalon, and reimbursements for conference travel expenses from Vanda

Pharmaceuticals. His institution has received equipment donations or other support from Optalert, Compumedics, Philips Lighting and Tyco Healthcare. He has also served as an expert witness and consultant to shift work organisations. Mark Howard has undertaken consultancy work for the National Transport Commission and Victoria Police, and has received research grants and equipment support from ResMed Foundation, CRCMining and Sleep Diagnostics. He is a participant in the Cooperative Research Centre for Alertness, Safety and Productivity and a member of the boards of the Australasian Sleep Association and the Institute for Breathing and Sleep, which receives royalties from Prevention Express.

Provenance: Commissioned by supplement editors; externally peer reviewed.

1 Vyas MV, Garg AX, lansavichus AV, et al. Shift work and vascular events: systematic review and meta-analysis. BMJ 2012; 345: e4800.

2 Rajaratnam SM, Arendt J. Health in a 24-h society. Lancet 2001; 358: 999-1005.

3 Barger LK, Ogeil RP, Drake CL, et al. Validation of a questionnaire to screen for shift work disorder. Sleep 2012; 35: 1693-1703.

4 Morris CJ, Yang JN, Scheer FA. The impact of the circadian timing system on cardiovascular and metabolic function. Prog Brain Res 2012; 199: 337-358.

5 Dawson D, Reid K. Fatigue, alcohol and performance impairment. Nature 1997; 388: 235.

6 Barger LK, Lockley SW, Rajaratnam SM, Landrigan CP. Neurobehavioral, health, and safety consequences associated with shift work in safety-sensitive professions. Curr Neurol Neurosci Rep 2009; 9: 155-164. 


\section{Sleep disorders}

7 Killick R, Banks S, Liu PY. Implications of sleep restriction and recovery on metabolic outcomes. J Clin Endocrinol Metab 2012; 97: 3876-3890.

8 Klerman EB, Dijk DJ. Interindividual variation in sleep duration and its association with sleep debt in young adults. Sleep 2005; 28: 1253-1259.

9 Cohen DA, Wang W, Wyatt JK, et al. Uncovering residual effects of chronic sleep loss on human performance. Sci Transl Med 2010; 2: 14ra3.

10 Rajaratnam SM, Barger LK, Lockley SW, et al. Sleep disorders, health, and safety in police officers. JAMA 2011; 306: 2567-2578.

11 Di Milia L, Waage S, Pallesen S, Bjorvatn B. Shift work disorder in a random population sample--prevalence and comorbidities. PLOS One 2013; 8: e55306.

12 Howard ME, Desai AV, Grunstein RR, et al. Sleepiness, sleep-disordered breathing, and accident risk factors in commercial vehicle drivers. Am J Respir Crit Care Med 2004; 170: 1014-1021.

13 Gold DR, Rogacz S, Bock N, et al. Rotating shift work, sleep, and accidents related to sleepiness in hospital nurses. Am J Public Health 1992; 82: 1011-1014.

14 Wright KP Jr, Bogan RK, Wyatt JK. Shift work and the assessment and management of shift work disorder (SWD). Sleep Med Rev 2013; 17: 41-54.

15 Akerstedt T, Fredlund P, Gillberg M, Jansson B. A prospective study of fatal occupational accidents - relationship to sleeping difficulties and occupational factors. J Sleep Res 2002; 11: 69-71.

16 Connor J, Norton R, Ameratunga S, et al. Driver sleepiness and risk of serious injury to car occupants: population based case control study. BMJ 2002 324: 1125.

17 Mitler MM, Carskadon MA, Czeisler CA, et al. Catastrophes, sleep, and public policy: Consensus report. Sleep 1988; 11: 100-109.

18 Czeisler CA, Moore-Ede M, Coleman RM. Rotating shift work schedules that disrupt sleep are improved by applying circadian principles. Science 1982; 217: 460-462.

19 Straif K, Baan R, Grosse Y, et al. Carcinogenicity of shift-work, painting, and fire-fighting. Lancet Oncol 2007; 8: 1065-1066.

20 Davila EP, Florez H, Fleming LE, et al. Prevalence of the metabolic syndrome among US workers. Diabetes Care 2010; 33: 2390-2395.

21 Karlsson B, Alfredsson L, Knutsson A. Total mortality and cause-specific mortality of Swedish shift- and dayworkers in the pulp and paper industry in 1952-2001. Scand J Work Environ Health 2005; 31: 30-35.
22 Smith-Coggins R, Rosekind MR, Buccino KR, et al. Rotating shiftwork schedules: can we enhance physician adaptation to night shifts? Acad Emerg Med 1997; 4: 951-961.

23 Fahrenkopf AM, Sectish TC, Barger LK, et al. Rates of medication errors among depressed and burnt out residents: prospective cohort study. BMJ 2008; 336: 488-491.

24 Culpepper $\mathrm{L}$. The social and economic burden of shift-work disorder. J Fam Pract 2010; 59 (1 Suppl): S3-S11.

25 Buysse DJ. Insomnia. JAMA 2013; 309: 706-716.

26 Monchesky TC, Billings BJ, Phillips R, Bourgouin J. Zopiclone in insomniac shiftworkers. Evaluation of its hypnotic properties and its effects on mood and work performance. Int Arch Occup Environ Health 1989; 61: 255-259.

27 Czeisler CA, Walsh JK, Roth T, et al. Modafinil for excessive sleepiness associated with shift-work sleep disorder. N Engl J Med 2005; 353: 476-486.

28 Ker K, Edwards PJ, Felix LM, et al. Caffeine for the prevention of injuries and errors in shift workers. Cochrane Database Syst Rev 2010; (5): CD008508.

29 Carrier J, Fernandez-Bolanos M, Robillard R, et al. Effects of caffeine are more marked on daytime recovery sleep than on nocturnal sleep. Neuropsychopharmacology 2007; 32: 964-972.

30 Ruggiero JS, Redeker NS. Effects of napping on sleepiness and sleep-related performance deficits in night-shift workers: a systematic review. Biol Res Nurs 2013; Feb 13 [Epub ahead of print].

31 Wertz AT, Ronda JM, Czeisler CA, Wright KP Jr. Effects of sleep inertia on cognition. JAMA 2006; 295: 163-164.

32 Signal TL, van den Berg MJ, Mulrine HM, Gander PH. Duration of sleep inertia after napping during simulated night work and in extended operations. Chronobiol Int 2013; 29: 769-779.

33 Rajaratnam SMW, Cohen DA, Rogers NL. Melatonin and melatonin analogues. Sleep Med Clin 2009; 4: 179-193.

34 Boivin DB, James FO. Light treatment and circadian adaptation to shift work. Ind Health 2005; 43: 34-48.

35 Barger LK, Wright KP Jr, Hughes RJ, Czeisler CA. Daily exercise facilitates phase delays of circadian melatonin rhythm in very dim light. Am J Physiol Regul Integr Comp Physiol 2004; 286: R1077-R1084. 